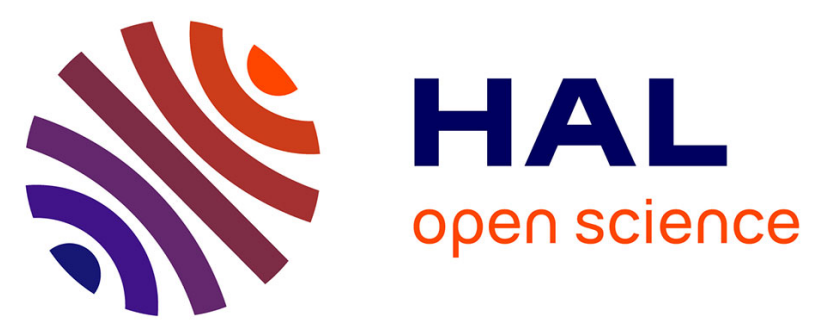

\title{
Séquentialité de la perception haptique et opérations descriptives: analyse qualitative du discours de trente locuteurs francophones sur quatre objets courants
} Bertrand Verine

\section{- To cite this version:}

Bertrand Verine. Séquentialité de la perception haptique et opérations descriptives: analyse qualitative du discours de trente locuteurs francophones sur quatre objets courants. Teresa Muryn, Salah Mejri. Linguistique du discours: de l'intra- à l'interphrastique, 8, Peter Lang, pp.219-231, 2015, Études de linguistique, littérature et art, 978-3-631-64945-9. 10.18463/verine.001 . hal-01314725

\author{
HAL Id: hal-01314725 \\ https://hal.science/hal-01314725
}

Submitted on 11 May 2016

HAL is a multi-disciplinary open access archive for the deposit and dissemination of scientific research documents, whether they are published or not. The documents may come from teaching and research institutions in France or abroad, or from public or private research centers.
L'archive ouverte pluridisciplinaire HAL, est destinée au dépôt et à la diffusion de documents scientifiques de niveau recherche, publiés ou non, émanant des établissements d'enseignement et de recherche français ou étrangers, des laboratoires publics ou privés. 


\title{
Séquentialité de la perception haptique et opérations descriptives : analyse qualitative du discours de trente locuteurs francophones sur quatre objets courants
}

\author{
Bertrand Verine, CNRS Université Paul-Valéry Montpellier, Praxiling (UMR 5267) \\ avec le soutien de la Fédération des Aveugles et Handicapés Visuels de France
}

[Ce travail a été présenté au $1^{\mathrm{er}}$ colloque international La Perception en langue et en discours, Varsovie, 24-26 avril 2014. Une version étendue a paru dans Teresa Muryn et Salah Mejri (éd.), 2015, Linguistique du discours : de l'intra- à l'interphrastique, Francfort-sur-le-Main : Peter Lang, p. 219-231. Les nombres intercalés dans le texte sans parenthèses ni crochets indiquent la pagination des segments communs avec la version papier.]

\section{Beaucoup de tripotages et d'empoignements, mais pas de réel toucher [...] on ne voit pas la douce circulation du toucher. (D. H. Lawrence, Croquis étrusques)}

Dans le vaste champ encore peu défriché de la perception en langue et en discours, les diverses formes de perception tactile occupent une place tout à fait périphérique. J'ai d'abord tenté de pallier ce manque en proposant pour le bicentenaire de Louis Braille un concours d'écriture sur le thème Dire le non-visuel. Ce premier corpus m'a notamment permis de falsifier l'argument a priori de l'indicibilité du toucher ${ }^{1}$ (Verine, 2014b). Ses verbalisations sont cependant demeurées plus rares et plus brèves que celles de l'ouïe et de l'olfaction. J'ai donc sollicité les psychologues Édouard Gentaz et Yvette Hatwell, spécialistes de ce système perceptif, pour recueillir de nouvelles données susceptibles d'être étudiées aussi bien selon les méthodes de la psychologie expérimentale que selon celles des sciences du langage.

C'est ainsi qu'en partenariat avec le laboratoire de Psychologie et NeuroCognition (UMR 5105 CNRS Université Grenoble 2), a été constitué le corpus DVPH (Description verbale et perception haptique ${ }^{2}$, Verine, Chauvey, Hatwell \& Gentaz, 2013). Il s'agit d'énoncés oraux non préparés produits en entretien semi-directif par trois groupes de locuteurs : dix personnes voyantes, ordinairement soumises

220

au primat du visuel, mais provisoirement empêchées de voir par un bandeau; dix personnes aveugles tardives ${ }^{3}$, obligées par leur cécité acquise à reconfigurer leurs habitudes perceptives ; et dix personnes aveugles congénitales qui, n'ayant jamais vu ou ayant cessé de voir avant l'âge de 1 an, ont structuré leur cognition via les seules ressources des autres systèmes perceptifs. À chacun de ces trente informateurs, interviewés séparément, nous avons demandé de «percevoir et décrire » quatre petits objets de la vie courante ${ }^{4}$, qui leur ont été remis dans

\footnotetext{
${ }^{1}$ Selon plusieurs chercheurs, certaines, voire toutes les langues ne disposeraient pas des éléments nécessaires à l'expression des perceptions tactiles, olfactives et gustatives. L'étude de corpus authentiques montre que, dès lors qu'ils se le proposent ou qu'on les y invite, des locuteurs même non experts trouvent des moyens variés et parfois innovants de verbaliser ces perceptions auxquelles l'interdiscours dominant ne fournit pas de prêt-à-dire.

${ }^{2}$ Cet adjectif issu du grec a été emprunté à l'anglais par les psychologues pour spécifier la perception tactile active via la ou les mains.

${ }^{3}$ Nous avons considéré comme aveugles tardives les personnes ayant perdu la vue après l'âge de 6 ans et depuis une durée supérieure à 5 années. Bien que l'enquête se soit étalée sur 19,5 mois (du 12.5.2010 au 26.12.2011), et pour ne pas la prolonger outre mesure, nous avons dû nous résoudre à quelques irrégularités. Ainsi avons-nous six femmes et quatre hommes aveugles de naissance pour quatre femmes et six hommes aveugles tardifs. Le dernier groupe se distingue également des deux autres par une moyenne d'âge supérieure et par un niveau scolaire inférieur.

${ }^{4}$ Ces quatre tâches à la fois haptiques et verbales étaient précédées par quatre tâches purement verbales, dont je ne traiterai pas ici, consistant à décrire de mémoire la femme et l'homme ayant assumé leur éducation.
} 
un ordre tiré au sort à chaque fois : un ancien téléphone portable (déconnecté), un porte-clés garni de quatre clés de taille et de forme différentes, une brosse à dents et un porte-monnaie (vide).

Après avoir mis en relation quelques traits pertinents de la perception haptique selon les psychologues (1.1.) et de la séquence descriptive selon les linguistes (1.2.), je présenterai les premiers résultats de l'analyse qualitative du corpus concernant la désignation des objets à décrire (2.1.), de leurs parties et de leurs propriétés (2.2.).

\section{Séquentialité de l'exploration manuelle active et des opérations descriptives $[\ldots]$ 222}

Je ne mentionnerai ici que les traits déterminants pour l'analyse du corpus $D V P H$, en renvoyant pour une problématisation d'ensemble aux nombreux ouvrages de Jean-Michel Adam (notamment 1997). Le premier de ces traits est que - contrairement à la narration, à l'explication ou à l'argumentation - la description ne constitue pas une séquence au sens fort de suite ordonnée de propositions, mais au sens affaibli où le locuteur y accomplit un ensemble d'opérations visant à permettre au destinataire de se représenter l'entité décrite. En particulier, toute nomination comporte une dimension descriptive dans la mesure où, par son choix lexical, le locuteur présuppose que l'entité nommée présente au moins un des traits définitoires de la catégorie à laquelle il l'assimile. On ne pourra donc commencer à parler de séquence à visée descriptive qu'à partir du moment où le locuteur accompagne son acte de nomination d'au moins une autre opération descriptive spécifiant l'entité considérée par rapport aux autres représentants de la même catégorie. Ainsi, dans l'exemple [1], notre informateur le moins prolixe ne produit pas de séquence descriptive puisqu'il ne spécifie la nomination c'est un portefeuille que par l'expansion avec plusieurs poches, qui peut discriminer l'objet par rapport à certains porte-monnaies, mais ne le distingue pas de tous les autres portefeuilles. Dans l'exemple [2], en revanche, ce même locuteur ébauche une séquence minimale en faisant suivre c'est une brosse à dents des caractérisations un manche, des poils et assez long ${ }^{5}$ :

[1] ça c'est / ça c'est c'est simplement ben un portefeuille avec euh avec quoi avec euh pluplusieurs euh plusieurs poch(e)s quoi puis euh ${ }^{\circ} h^{\circ} h^{\circ} h$ donc euh / ouais c'est un port(e)monnaie ou u:n portefeuille [LAC18S8 intégrale] ;

223

[2] non mais c'est une: une brosse à dents donc u::n manche avec des des des poils c'est assez long euh ${ }^{\circ} \mathrm{h}$ ouais c'est un(e) brosse à dents [LAC18S5 intégrale].

On parle d'opération d'ancrage lorsque la désignation de l'entité à décrire, ou hyperthème de la description, se fait a priori, c'est-à-dire en début de séquence, avant le développement de ses spécifications, comme en [2]. On parle d'opération d'affectation lorsque la désignation de l'hyperthème se fait a posteriori, en cours ou en fin de séquence, après le développement de certaines ou de toutes ses spécifications, comme en [3] où les caractérisations assez gros, un écran et des touches précèdent la nomination c'est un téléphone portable:

\footnotetext{
${ }^{5}$ La source des exemples est indiquée selon le schéma [Locuteur, numéro d'identification, Séquence, numéro d'ordre] ; LAC correspond aux locuteurs aveugles congénitaux, LAT aux locuteurs aveugles tardifs et LYB aux locuteurs voyants aux yeux bandés. Je remercie les quatre stagiaires qui ont finalisé la mise en forme du corpus : Alice Blanc et Anna Prat pour l'anonymisation et le séquençage des vidéos, Marion Mezen et Cécile Prouhèze pour leur transcription. Conventions de transcription : [A] réfère à l'enquêtrice et [B] à l'interviewé/e ; [-] note l'aphérèse ou l'apocope d'une ou plusieurs syllabes ; [O] note l'élision d'un phonème ; [:] marque l'allongement d'une voyelle ; [/] indique la pause silencieuse ; $\left[{ }^{\circ} \mathrm{h}\right]$ note les inspirations audibles.
} 
[3] alors ça: pour moi ça: donc ça c'est donc euh ${ }^{\circ} \mathrm{h}$ euh c'est c'est gros assez gro:s euh un écran des touches c'est un téléphon(e) portable / on peut arrêter là [LAC18S6 intégrale].

Parmi les opérations visant à spécifier l'hyperthème, je ne retiendrai ici que l'aspectualisation ${ }^{6}$, qui peut s'accomplir par qualification, c'est-à-dire par attribution à l'entité de diverses propriétés (assez long en [2], assez gros en [3]) et/ou par fragmentation, c'est-àdire par décomposition de l'entité en différentes parties (manche et poils en [2], écran et touches en [3]). Je soulignerai que les aspects jugés les plus pertinents par le locuteur peuvent faire l'objet d'une opération de sous-thématisation qui les constituera en sous-ensembles à leur tour spécifiés par qualification et/ou fragmentation. Il en va notamment ainsi, dans $D V P H$, pour les trois parties dont le toucher ne peut détecter qu'une forme générique qui suscite des hypothèses, la vue étant indispensable pour préciser leur catégorisation : il s'agit de la marque inscrite sur la brosse à dents, ainsi que de l'effigie de Tintin et d'un dragon brodée sur le porte-monnaie, toutes deux trop denses pour être interprétables par le doigt, et plus fréquemment, du bonhomme dessiné sur le porte-clés, dont le support très schématique demeure ambigu pour la main, et qui inclut le bouton-poussoir d'une commande à distance. Pour exemple, je juxtapose en [4] l'ouverture d'une séquence où la locutrice retarde explicitement l'aspectualisation du porte-clés au profit de celle de chacune des quatre clés, et sa chute où elle boucle son propos en revenant sur le sous-thème différé :

[4] ok alors c'est u::n porte-clés ${ }^{\circ} \mathrm{h}$ en forme de:: c'(es)t un(e) grand(e) question à laquell(e) je répondrai plus tard ${ }^{\circ} \mathrm{h}[\ldots .$.$] eu:h / le porte-clés en lui-même euh j(e) suis pas trop décidée sur c(e) que ça peut êt(re) p(eu)t-être$ 224

un genr(e) de personnage ou: que(l)qu(e) chos(e) comm(e) ça / eu::h voilà en gros $c(e)$ que $j(e)$ peux dire [LAC4S5 attaque et chute].

Enfin, la séquence descriptive peut passer par et/ou aboutir à une opération de reformulation, particulièrement intéressante dans les cas où elle recatégorise l'hyperthème par rapport à sa première nomination. Ainsi, la séquence LAT12S6 s'ouvre sur la nomination alors c'est un étui, mais dès la fin du premier quart, le locuteur actualise la reformulation ben oui c'est un porte-monnaie. De même, la locutrice de la séquence LAT14S7 s'appuie d'abord sur la nomination alors ça c'est une c'est une pochette, réitérée à trois reprises, mais elle introduit dans le dernier tiers la reformulation et à l'arrière du de ce porte-monnaie, sur laquelle elle clôt sa description.

C'est sur ces bases que je voudrais tenter de réexaminer le double paradoxe suivant (pointé dans Verine, 2014b): d'une part, la rhétorique occidentale a explicité des procédures permettant de structurer en séquences la représentation des perceptions visuelles, qui sont le plus souvent synthétiques et immédiates; d'autre part, la verbalisation des perceptions haptiques est réputée difficile (voire impossible), alors que la séquentialité d'abord analytique puis configurationnelle de ce système perceptif semble a priori plus compatible avec la progressivité des opérations descriptives.

\section{La perception haptique à l'épreuve de la verbalisation et réciproquement 2.1. Ancrage spontané $v s$ affectation contrainte}

Il convient d'abord de minimiser, sans l'écarter totalement, une circonstance aggravante pour la difficulté de certains locuteurs : notre dispositif expérimental ne recrée pas la situation la plus prototypique de production d'une séquence descriptive, celle où un locuteur fragmente et qualifie un hyperthème qu'il connaît (tant soit peu) pour un destinataire qui ne le connaît pas

\footnotetext{
${ }^{6}$ Les diverses opérations de mise en relation (spatiale, temporelle, analogique et dialogique) sont moins systématiquement attestées par le corpus.
} 
ou ne l'a pas présent à l'esprit. Dans $D V P H$, au contraire, l'enquêtrice possède d'emblée de nombreuses informations sur les quatre objets et demande aux interviewés de lui fournir celles qu'ils identifient sans préparation. Je soulignerai que cette forme d'inversion de l'asymétrie interlocutive peut apparaître dans certaines interactions spontanées, telles que les indications d'itinéraire par téléphone où le guide doit s'appuyer sur les éléments que découvre la personne qu'il renseigne. Notre protocole demeure donc écologique.

La comparaison avec un autre contexte descriptif également non prototypique, mais très fréquent, permet de proposer une seconde explication

225

d'un rendement supérieur : la description concomitante à l'observation semble d'autant plus aisée que l'identification de l'hyperthème est préalablement accomplie par un ancrage précis et assuré, ce que l'exploration haptique ne garantit pas toujours. De fait, si le reportage en direct (principalement basé sur la vue) est un art parfois délicat, mais pratiqué par de nombreux journalistes de radio, c'est que l'hyperthème et certaines de ses parties leur sont déjà connus : protocole d'une cérémonie officielle ou règles d'un sport, nom et statut des participants, par exemple. Or, dans le cas de la description d'objets, «l'appréhension morcelée et très séquentielle » que procure le toucher n'aboutit pas toujours rapidement au « travail mental d'intégration et de synthèse » (Gentaz et al., 2009:3) permettant d'ancrer la description sur une catégorisation certaine ${ }^{7}$.

Il en va notamment ainsi pour le téléphone portable proposé lors de notre expérience, en raison de sa forme désuète, du marquage ambigu de sa touche 5 , de l'absence de dispositif d'ouverture-fermeture et, surtout, de la présence paradoxale du support d'une antenne arrachée. Sur nos trente informateurs, dix-sept identifient d'emblée la vétusté de l'appareil, tandis que quatre ne verbalisent ni cette propriété ni la partie support d'antenne. En revanche, quatre autres actualisent des ancrages extrêmement modalisés et/ou explicitent la mise en débat par ou, puis la confirmation par oui de la catégorisation choisie, comme en [5], tandis que deux produisent des séquences intégralement problématisantes et irrésolues (à la fois, entre les deux, je sais pas), dont je rapproche sous [6] et [7] les extraits les plus révélateurs :

[5] ho c'est un(e) télécommand(e) ça / ah oui / ou un téléphone ou un téléphone portable / oui c'est un téléphone portable / alors oui puisque là là y a le joys- les chiffr(e)s un deux trois $\mathrm{j}(\mathrm{e})$ suis en train $\mathrm{d}(\mathrm{e})$ chercher le cinq si $\mathrm{y}$ a un point mais // ouais il est pas très apparent / i(l) devrait être ici / oui donc c'est un téléphone portable [...] [LAT12S5 attaque] ;

[6] Y'a qu'est-ce que je trouve c'est un un deux trois quatre une en quelque sorte c'est une télécommande ou bien c'est peut-être bien un téléphone ça peut ça peut être à la fois je pense c'est un téléphone un téléphone portable [...] je je pense que c'est une télécommande à mon avis ou bien c'est ou bien une télécommande ou bien un téléphone entre les deux mais une télécommande normalement elle devrait avoir plusieurs touches encore beaucoup plus de touches que celle-là [LAT15S5 attaque et chute] ;

226

[7] [...] après après quoi dire de plus ça me perturbe parce que je sais pas si c'est un téléphone j'arrive pas à enlever le la batterie donc je sais pas ça ressemble à un téléphone ou alors un ou un jouet pour enfant [...] voilà je sais pas si ça doit être un téléphone portable mais enfin c'est un téléphone portable si c'est sûr de toutes façons après que ce soit un vrai ou un faux je sais pas voilà [...] [LYB22S5 cours].

Seul notre locuteur 30 utilise manifestement la procédure d'affectation comme une stratégie discursive visant à retarder le moment de nommer chacun des objets, y compris, en [8], le plus facile à identifier, la brosse à dents. Mais surtout, l'exemple [9] montre qu'il énonce, ainsi que les deux derniers descripteurs du téléphone, une affectation réfutée permettant d'exclure une nomination concurrente, alors même qu'elle n'a pas été mentionnée jusque-là ; il semble donc

\footnotetext{
${ }^{7}$ Nous n'avons malheureusement pas pensé à soumettre les mêmes objets à un quatrième groupe d'informateurs qui les aurait perçus et décrits en pouvant recourir à leurs yeux ; mais S'agissant d'objets usuels, il paraît très probable que l'ancrage et la fragmentation n'auraient pas posé de problème.
} 
qu'on ne puisse expliquer la survenue inopinée de telles réfutations que parce que la possibilité d'une autre catégorisation entrave l'avancée du processus descriptif :

[8] A68- donc c'est quelque chos(e) d'assez léger /// que je reconnais mais que je n(e) nomm(e) pas encor(e) dont je n(e) dis pas c(e) que c'est

B69- ah ben vous pouvez hein

A70- donc ça ressemble à un(e) brosse à dents [...] [LYB30S5] ;

[9] donc c'est plutôt un objet un peu plus lourd que les autres / euh en plastique // on dirait un téléphone y aurait un écran là ici des touches / eu:h ici y aurait une antenne qui aurait été enlevée par exemple [...] -fin on on je j'ai pas la sensation que ce soit une télécommande [...] [LYB30S8 attaque et cours] ;

[10] [...] c'est quand mêm(e) pas un(e) télécommande de de télévision ou de quoi qu(e) ce soit [...] [LAT11S7 cours];

[11] [...] -fin eu:h ça peut pas être un(e) calculatrice ${ }^{\circ} \mathrm{h}[\ldots]$ [LAT19S8 cours].

Tous ces éléments tendent à prouver que, si l'affectation est bien attestée à l'écrit et dans l'oral préparé, elle reste rare en interaction spontanée et ne correspond pas de manière univoque à la verbalisation des tâches d'identification accomplies par le locuteur : elle consiste en réalité à retarder l'explicitation d'une catégorisation que le locuteur doit avoir effectuée, tacitement ou non, pour pouvoir développer efficacement les autres opérations descriptives, en particulier celle de fragmentation.

\subsection{Aspectualisation intuitive vs méthodique}

La difficulté - souvent alléguée et parfois réelle - de fonder la description d'un objet sur sa perception haptique serait donc imputable à la séquentialité du toucher lui-même dans les cas où l'hyperthème

227

apparaît difficile à catégoriser de prime abord. Cependant, l'examen de la fragmentation et de la qualification permet de pondérer entre elles d'autres explications qui tiennent aux habitudes discursives et praxiques des locuteurs, et dépendent donc dans une large mesure de facteurs psycho-sociaux. Un premier groupe de raisons pourrait tenir à l'habitude inégalement partagée entre les locuteurs de recevoir et de produire tous types de séquences descriptives, voire à l'inégalité de leur formation scolaire. Ainsi est-il vrai que notre descripteur le moins loquace (supra [1], [2] et [3]) a arrêté sa formation au BEP. Mais d'autres locuteurs moins scolarisés (LAT2, LAT10, LAT11) parviennent à développer des descriptions pertinentes et, symétriquement, certains informateurs ayant un niveau d'étude très supérieur (comme LYB29, titulaire d'un Master 2) rencontrent des difficultés pour fragmenter et/ou pour qualifier un ou plusieurs des quatre objets. Je ne rappelle ici que pour mémoire mon hypothèse initiale (Verine, 2007), imputant la rareté et la brièveté des notations tactiles à la pénurie d'exemples dans les genres du discours littéraires et médiatiques socialement valorisés : la consigne et les objets proposés dans l'enquête $D V P H$ ont eu précisément pour but de neutraliser le plus possible ce facteur.

Une explication plus convaincante semble devoir être cherchée dans la plus ou moins grande habitude d'interpréter les informations tactiles. De fait, bien que Chauvey et al. (2012) ne constatent pas, en termes statistiques, de corrélation significative entre le facteur groupe et le nombre de mots référant au toucher ou à des perceptions multimodales, une analyse détaillée de l'opération de fragmentation atteste que les trois groupes ne distinguent pas le même nombre de parties à l'intérieur des deux objets les plus complexes. Ainsi, pour le portemonnaie, six locuteurs aveugles congénitaux identifient trois poches et un en évoque plusieurs, contre quatre aveugles tardifs et aucun voyant aux yeux bandés ; réciproquement, deux locuteurs aveugles congénitaux mentionnent deux poches seulement, contre cinq aveugles tardifs et tous les voyants aux yeux bandés; enfin, deux locuteurs aveugles, l'un congénital et l'autre tardif, ne procèdent pas à cette fragmentation. De même, en ce qui 
concerne les clés, huit locuteurs aveugles congénitaux en comptent quatre, contre neuf aveugles tardifs et six voyants seulement. De même encore, six locuteurs aveugles congénitaux repèrent la présence du bouton poussoir d'une commande dans le porte-clés, contre trois aveugles tardifs et quatre voyants (voir infra annexe 1).

Malgré quelques irrégularités de détail, ces constatations indiquent globalement que les locuteurs aveugles congénitaux, contraints depuis toujours à aiguiser leur sensibilité tactile pour percevoir les objets, procèdent à une fragmentation plus précise que les deux autres groupes. Cela confirme les observations de Galiano et al. (2012), et se trouve corroboré par la finesse de qualification de la brosse à dents elle-même,

228

objet le plus simple de notre corpus, sur lequel ces locuteurs repèrent plus souvent que les autres l'inscription de la marque et les variations de texture du plastique. La différence d'entraînement à l'exploration haptique peut aussi être corrélé avec le fait que les neuf locuteurs à qui l'identification de notre téléphone portabe pose problème (supra 2.1.) se répartissent en un aveugle congénital seulement, contre cinq aveugles tardifs et trois voyants aux yeux bandés ${ }^{8}$.

Cette explication apparemment robuste et objective doit cependant être combinée avec un autre facteur que révèle l'étude de l'aspectualisation de nos quatre objets par les trente locuteurs : la recherche méthodique (ou non) de propriétés tangibles et d'inférences praxiques. De fait, si les locuteurs aveugles tardifs apparaissent faire preuve d'une moindre sensibilité tactile, ils s'avèrent rationaliser davantage leur exploration. Ainsi sont-ils huit sur dix à verbaliser, lors de la description du téléphone, le marquage en relief de la touche 5 , contre cinq aveugles congénitaux seulement et un unique voyant. De même sont-ils sept à structurer l'aspectualisation des clés selon leur taille (dont six par ordre croissant, c'est-à-dire en commençant par la moins facile à identifier) contre six aveugles congénitaux et cinq voyants (dont quatre seulement, dans ces deux groupes, suivent l'ordre progressif). Ils sont aussi plus nombreux à caractériser trois ou quatre des clés par leurs contextes potentiels d'usage (cadenas, boîte aux lettres, porte intérieure et voiture): six aveugles tardifs contre trois congénitaux et quatre voyants. Ils sont à nouveau six à qualifier les poches du porte-monnaie par leurs destinations (billets, pièces et parfois cartes), contre quatre aveugles congénitaux et cinq voyants (voir infra annexe 2).

Tout se passe donc comme si les locuteurs aveugles congénitaux et voyants aux yeux bandés abordaient l'opération d'aspectualisation de manière plus spontanée, en se fiant avant tout à leurs intuitions. Sans doute, peut-on corréler cette tendance avec mes résultats précédents sur la modalisation de ces séquences de description d'objets (Verine, 2014a), montrant que les premiers assument notre protocole comme une simple mise en spectacle de leur praxis ordinaire, tandis que les seconds le considèrent comme un test ludique dont le résultat n'engage pas sérieusement leur compétence. En revanche, les locuteurs aveugles tardifs, dont les marques modalisantes et, ici même, les difficultés d'ancrage attestent qu'ils sont davantage déstabilisés par la consigne de « percevoir et décrire »,

229

recourent plus systématiquement à une procédure concertée d'exploration et de verbalisation. Ce résultat appelle une recherche beaucoup plus vaste afin de déterminer quelle est la part d'éventuels facteurs neurocognitifs susceptibles d'expliquer que la perte, même ancienne, de la vue modifie durablement les activités de catégorisation et de caractérisation, et quelle est la part de facteurs psychosociaux tels que les techniques compensatoires enseignées par les rééducateurs et les discours contribuant à cette réadaptation.

\footnotetext{
${ }^{8}$ J'ai également montré (Verine 2014a) que ces locuteurs sont de très loin ceux qui modalisent le moins souvent l'opération d'ancrage : 7 occurrences seulement sur les 40 descriptions produites par ce groupe, contre 14 sur 40 pour les locuteurs voyants et 16 sur 40 pour les aveugles tardifs.
} 
Au total, malgré l'étroitesse du corpus et la faiblesse de certains écarts de fréquence, la convergence de ces éléments paraît suffisante pour tirer trois conclusions qui précisent les observations de Galiano et al. (2012) sur d'autres discours et affinent les résultats statistiques obtenus par Chauvey et al. $(2012,2013)$ sur ce même corpus $D V P H$. La première est que la séquentialité du toucher manuel actif s'avère compatible avec celle des opérations de fragmentation et de qualification, permettant sans conteste d'actualiser des descriptions pertinentes et détaillées de tous les objets proposés. La seconde est que, par rapport à l'immédiateté synthétique de la vue, cette séquentialité impose aux locuteurs un fort désavantage pour l'identification initiale de l'hyperthème. La troisième est que cette difficulté et sa solution ne résident pas seulement dans le degré de la sensibilité haptique, mais dans le développement d'une stratégie exploratoire et du traitement langagier des indices recueillis.

$\mathrm{Au}$ niveau applicatif, de telles données devraient conduire les rééducateurs de personnes aveugles à les entraîner plus systématiquement à l'exploration tactile en valorisant la verbalisation de leurs perceptions, à plus forte raison quand elles perdent la vue tardivement. $\mathrm{Au}$ niveau linguistique, la redéfinition de l'affectation comme un ancrage différé pour le locuteur lui-même et la fréquence de la qualification des parties par leurs usages potentiels montrent l'intérêt d'enraciner les modèles forgés sur les textes écrits dans la réalité ordinaire des pratiques langagières, comme l'ont fait les narratologues pour le récit dans les années 1980, et comme a commencé à le faire l'équipe de Danièle Dubois (2009) pour l'ensemble des propriétés perceptives.

\section{Références}

Adam, J.-M. (1997). Les Textes : types et prototypes. Paris : Nathan.

Chauvey, V., Hatwell, Y. \& Gentaz, É. (2013). Verbalisme, références aux modalités sensorielles non-visuelles et communication verbale entre adultes aveugles et voyants. Médiation et information, 36, 155-166.

Chauvey, V., Hatwell, Y., Kaminski, G., Gentaz, É. \& Verine, B. (2012). Lexical references to sensory modalities in verbal descriptions of people and objects by congenitally blind, late blind and sighted adults. PLOS ONE, 7(8), e44020 (DOI 10.1371/journal.pone.0044020).

Dubois, D. dir. (2009). Le Sentir et le dire. Concepts et méthodes en psychologie et en linguistique cognitive. Paris : L'Harmattan.

230

Galiano, A.-R., Portalier, S., Baltenneck, N., Griot, M. \& Poussin, M. (2012). Étude pragmatique des compétences référentielles des personnes aveugles. Bulletin de psychologie, 518, 129-139.

Gentaz, É., Bara, F., Palluel-Germain, R., Pinet, L. \& Hillairet de Boisferon, A. (2009). Apports de la modalité haptique manuelle dans les apprentissages scolaires (lecture, écriture et géométrie). In Cognito, 3(3), 1-38.

Verine, B. (2007). À la recherche du point de vue aveugle. Voir barré, 34-35, 99-115.

Verine, B. (2014a). Les modalisations d'(in)certitude et d'(im)précision comme instruments d'analyse qualitative d'un objet de discours à la marge: les perceptions tactiles. Cahiers de praxématique 62, www.praxematique.revues.org/

Verine, B. (2014b). Pour une approche des perceptions tactiles en discours. in B. Verine (dir.) Dire le nonvisuel: approches pluridisciplinaires des discours sur les perceptions autres que la vue (-). Liège : Presses Universitaires de Liège, 143-163.

Verine, B., Chauvey, V., Hatwell, Y. \& Gentaz, É. (2013). Description verbale et perception haptique (corpus de trente vidéos d'expériences). Montpellier : Praxiling.

Annexe 1 : taux de nomination de certaines parties pour chaque groupe de 10 locuteurs

\begin{tabular}{|l|l|l|l|}
\hline Fragmentation & Congénitaux & Tardifs & Yeux bandés \\
\hline Trois poches & 6 & 4 & 0 \\
\hline Quatre clés & 8 & 9 & 6 \\
\hline Poussoir & 6 & 3 & 4 \\
\hline
\end{tabular}

Annexe 2 : taux de désignation de certaines propriétés pour chaque groupe de 10 locuteurs 


\begin{tabular}{|l|l|l|l|}
\hline Qualification & Congénitaux & Tardifs & Yeux bandés \\
\hline Point sur le cinq & 5 & 8 & 1 \\
\hline Clés croissantes & 4 & 6 & 4 \\
\hline Usages des clés & 3 & 6 & 4 \\
\hline Usages des poches & 4 & 6 & 5 \\
\hline
\end{tabular}

231

Résumé. Ce travail vise à expliquer pourquoi la description tactile d'un objet est réputée difficile (voire impossible), alors que la séquentialité d'abord analytique puis configurationnelle du toucher devrait se prêter parfaitement à la progressivité des opérations descriptives. Dans ce but, on compare les descriptions orales de quatre objets courants produites sans préparation par trente locuteurs : dix aveugles congénitaux, dix aveugles tardifs et dix voyants aux yeux bandés. Les résultats montrent que la difficulté de fonder la description d'un objet sur sa perception tactile est imputable à la séquentialité du toucher luimême dans les cas où l'objet apparaît difficile à catégoriser de prime abord, car le locuteur doit avoir effectué cette catégorisation avant de développer efficacement les autres opérations descriptives. Cependant, la comparaison des parties et des propriétés distinguées par les trois groupes d'informateurs révèle que cette difficulté et sa solution résident avant tout dans le degré d'entraînement à la perception tactile, dans le développement d'une stratégie exploratoire et dans le traitement langagier des indices recueillis.

Mots clés : toucher, description, catégorisation, aspectualisation, cécité.

This work aims to explain why the haptic description of an object is considered arduous (or even impossible), despite the tact's sequentiality: when they perceive by the tact, the subjects begin analysing the objects and continue by configuring their mental image, which should perfectly allow progression of descriptive processes. In order to explain this paradox, we compare the oral descriptions of four familiar objects produced without preparation by thirty speakers: ten congenitally blind, ten late blind and ten blindfolded sighted persons. The results show that the difficulty to describe an object based on its haptic perception refers to the tact's sequentiality only when the object cannot be easily categorised initially, because the speaker needs this categorisation before efficiently processing the other descriptive operations. However, contrasting the parts and properties of the objects distinguished by the three informant groups, we find that this difficulty and its solution consist of training towards haptic perception, developing an exploratory strategy and turning the collected cues into language. Keywords : haptic perception, description, categorisation, aspectualisation, blindness. 